\title{
Improvement of Accuracy of Marker-Free Bronchoscope Tracking Using Electromagnetic Tracker Based on Bronchial Branch Information
}

\author{
Kensaku Mori ${ }^{1,2}$, Daisuke Deguchi², Takayuki Kitasaka ${ }^{2,3}$, \\ Yasuhito Suenaga $^{1,2}$, Yosihnori Hasegawa ${ }^{4,2}$, \\ Kazuyoshi Imaizumi ${ }^{4}$, and Hirotsugu Takabatake ${ }^{5}$ \\ ${ }^{1}$ Graduate School of Information Science, Nagoya University, \\ Furo-cho, Chikusa-ku, Nagoya, Aichi, 464-8603, Japan \\ kensaku@is.nagoya-u.ac.jp \\ ${ }^{2}$ Innovative Research Center for Preventive Medical Engineering, Nagoya University \\ ${ }^{3}$ Faculty of Management and Information Science, Aichi Institute of Technology \\ ${ }^{4}$ Graduate School of Medicine, Nagoya University \\ ${ }^{5}$ Sapporo Minami-Sanjyo Hospital
}

\begin{abstract}
This paper presents a study of tracking accuracy improvement of marker-free bronchoscope tracking using an electromagnetic tracking system. Bronchoscope tracking is an important function in a bronchoscope navigation system that assists a physician during bronchoscopic examination. Several research groups have presented a method for bronchoscope tracking using an ultra-tiny electromagnetic tracker (UEMT) that can be inserted into the working channel of a bronchoscope. In such a system, it is necessary to find the matrix $\mathbf{T}$ showing the relation between the coordinate systems of the CT image and the UEMT. This paper tries to improve the accuracy of this matrix by using not only the position information of the UEMT but also the orientation information. The proposed algorithm uses the running direction information of bronchial branches and the orientation information of the UEMT in the computation process of $\mathbf{T}$. In the experiments using a bronchial phantom, the tracking accuracy was improved from $2.2 \mathrm{~mm}$ to $1.8 \mathrm{~mm}$.
\end{abstract}

\section{Introduction}

A flexible bronchoscope (RB: real bronchoscope) is a tool used for observing the inside of the bronchus or performing needle biopsy on suspicious regions. In an examination using a bronchoscope, a physician inserts the bronchoscope while watching a TV monitor and then inserts needles or brushes to obtain tissues as specimens. However, the bronchus has a complex tree structure and a physician easily can get disoriented. For this reason, a bronchoscope navigation system guiding a physician to the desired location is strongly expected to be developed. This system used CT image taken prior to actual bronchoscopic examination as a road map. Bronchoscope tracking is quite important function in a bronchoscope navigation system. 


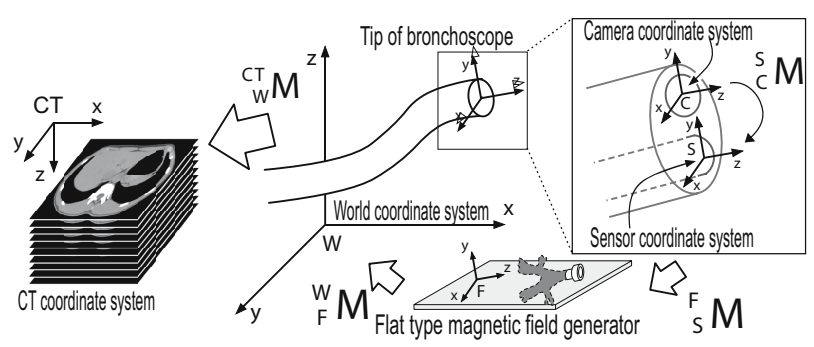

Fig. 1. Relation between each coordinate system

In the research of bronchoscope tracking, there are three types of studies: (a) tracking based on image registration, (b) tracking based on a positional sensor, and (c) a hybrid method. The former type tracks the bronchoscope by comparing virtual bronchoscopic (VB) images derived from the pre-operative $\mathrm{CT}$ image with the RB image [21. In this process, for each frame of RB, we generate a lot of VB images and find the most similar image to the current frame of the RB. This process is very time consuming. The other type is utilizing an ultra-tiny electromagnetic tracker (UEMT). In this case, a small sensing coil is attached to the tip of a bronchoscope. Wegner et al. projected the outputs of the UEMT onto the medial lines of the bronchus to achieve stable RB tracking [3]. However, the RB does not move along the medial lines of the bronchus. Also, these methods require placing several fiducial markers and need to measure the positions of these markers. The type (c) would be a good solution [5], but still the problem of fiducial markers is not solved.

As one of the solutions to solve the problem of type-(b) tracking, Deguchi et al. presented a paper that finds the transformation matrix, which shows the relation between the coordinated systems of the UEMT of the CT image, from the medial lines of the bronchus and the UEMT outputs 4. This process does not require measurements of fiducial locations, which is required in Wegner's method [3]. Also, it has become possible to track the $\mathrm{RB}$ when the $\mathrm{RB}$ does not exist on the medial lines. However, there is a possibility of a mis-corresponding finding between the medial lines and the UEMT outputs around branching points.

This paper solves the problem of Deguchi's method [4] by utilizing the running direction information of the bronchus and the orientation information of the UEMT. By this process, we try to avoid a miscorrespondence finding around branching points. Also, the thickness of a bronchial branch is considered here.

\section{Definition}

We use the CT coordinate system as the coordinate system of the VB. The relation between the position $\boldsymbol{p}_{C}$ in the RB camera coordinate system $C$ and the corresponding point $\boldsymbol{p}_{C T}$ in the CT coordinate system (Fig. 1) is expressed as

$$
\boldsymbol{p}_{C T}={ }_{W}^{C T} \mathbf{T}{ }_{F}^{W} \mathbf{T}_{S}^{F} \mathbf{T}_{C}^{k}{ }_{C} \mathbf{T} \boldsymbol{p}_{C}={ }_{W}^{C T} \mathbf{T}{ }_{S}^{W} \mathbf{T}_{C}^{k} \mathbf{T} \boldsymbol{p}_{C}={ }_{W}^{C T} \mathbf{T} \boldsymbol{p}_{W}^{k},
$$




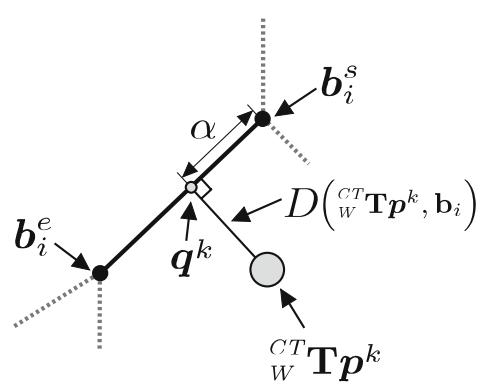

Fig. 2. Relation between RB tip ${ }_{W}^{C T} \mathbf{T} \boldsymbol{p}^{k}$ and bronchial branches $\mathbf{b}_{i}=\left\{\boldsymbol{b}_{i}^{s}, \boldsymbol{b}_{i}^{e}\right\}$

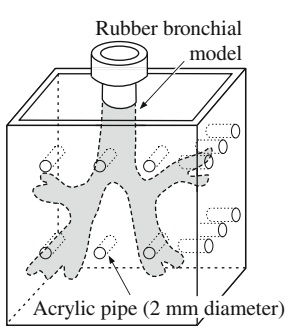

Fig. 3. Illustration of phantom used in experiments

where ${ }_{C}^{S} \mathbf{T}$ is the transformation from the RB camera coordinate system $C$ to the coordinate system $S$ of the sensor attached at the tip of the RB, ${ }_{S}^{F} \mathbf{T}$ from the coordinate system of $S$ to the UEMT coordinate system $F,{ }_{F}^{W T}$ from the UEMT coordinate system to the world coordinate system, and ${ }_{W}^{C T} \mathbf{T}$ from the world coordinate system to the CT coordinate system. We represent the coordinate of $\boldsymbol{p}_{C}$ in the world coordinate system as $\boldsymbol{p}_{W}$ and the position of $\boldsymbol{p}_{C}$ at time $k$ in the world coordinate system as $\boldsymbol{p}_{W}^{k}$.

The proposed method uses the medial lines of the bronchus region extracted from CT images to estimate ${ }_{W}^{C T} \mathbf{T}$. We represent the $i$-th bronchial branch as $\mathbf{b}_{i}=\left\{\boldsymbol{b}_{i}^{s}, \boldsymbol{b}_{i}^{e}\right\}$. Here, $\boldsymbol{b}_{i}^{s}$ and $\boldsymbol{b}_{i}^{e}$ are the start and the end points of the bronchial branch $\mathbf{b}_{i}$ on the CT image. The parent branch of $\mathbf{b}_{i}$ is represented by $\mathcal{P}\left(\mathbf{b}_{i}\right)$. A set of child branches $\mathbf{b}_{i}$ is expressed as $\mathcal{C}\left(\mathbf{b}_{i}\right)$. A set of all bronchial branches is represented by $\mathcal{B}=\left\{\mathbf{b}_{i} \mid i=1, \ldots, n\right\}$ ( $n=$ the number of bronchial branches.)

\section{Method}

We find the correspondence between the RB and the VB under the assumption that the RB tip moves along the medial lines of the bronchus. Hence, we need to find the transformation matrix showing the relation of both coordinate systems. This process corresponds computing the transformation matrix from the point $\boldsymbol{p}_{C}$ in the RB coordinate system to the corresponding point $\boldsymbol{p}_{C T}$ in the $\mathrm{CT}$ coordinate system in Eq. (11). Here, ${ }_{S}^{W} \mathbf{T}^{k}\left(={ }_{F}^{W} \mathbf{T}_{S}^{F} \mathbf{T}^{k}\right)$ shows the transformation from the sensor coordinate system $S$ to the world coordinate system and is calculated from the output of the UEMT. Also, the transformation matrix ${ }_{C}^{S} \mathbf{T}$ shows the relative relation between the $\mathrm{RB}$ camera and the sensor. This can be obtained when we attach the sensor at the tip of the bronchoscope. Therefore, it is possible to find the correspondence between the RB camera coordinate system and the CT coordinate system only by finding ${ }_{W}^{C T} \mathbf{T}$. We try to estimate ${ }_{W}^{C T} \mathbf{T}$ in this paper.

\subsection{Estimation of ${ }_{W}^{C T} \mathbf{T}$}

Measurement of the RB tip position and orientation by UEMT. Before estimation of the transformation matrix ${ }_{W}^{C T} \mathbf{T}$, we record the RB tip position $\boldsymbol{p}^{k}$ 
and the RB orientation (=view direction) $\boldsymbol{d}^{k}$, and obtain a set of RB tip positions and orientations $\mathcal{P}=\left\{\left(\boldsymbol{p}^{k}, \boldsymbol{d}^{k}\right)\right\}(k=1, \ldots, N)$ DThese are computed by the transformation matrix ${ }_{S}^{W} \mathbf{T}^{k}$ that is the output of the UEMT. While inserting the RB into the bronchus, we measure multiple $\left(\boldsymbol{p}^{k}, \boldsymbol{d}^{k}\right)$. For every $N$ measurement, we perform the procedure shown below and estimate ${ }_{W}^{C T} \mathbf{T}$.

(a) Selection of bronchial branch. For each viewpoint and view direction $\left(\boldsymbol{p}^{k}, \boldsymbol{d}^{k}\right) \in \mathcal{P}$ obtained in the previous section, we select a bronchial branch $\mathbf{b}_{*}$ corresponding to $\left(\boldsymbol{p}^{k}, \boldsymbol{d}^{k}\right)$. These $\left(\boldsymbol{p}^{k}, \boldsymbol{d}^{k}\right)$ and $\mathbf{b}_{*}$ will be used in correspondingpoint calculation in Section 3.1. For each bronchial branch $\mathbf{b}_{i}=\left\{\boldsymbol{b}_{i}^{s}, \boldsymbol{b}_{i}^{e}\right\}$. we measure the distance $D\left({ }_{W}^{C T} \mathbf{T}^{l} \boldsymbol{p}^{k}, \mathbf{b}_{i}\right)$ between ${ }_{W}^{C T} \mathbf{T}^{l} \boldsymbol{p}^{k}$ and $\mathbf{b}_{i}$ by

$$
\begin{gathered}
D\left({ }_{W}^{C T} \mathbf{T} \boldsymbol{p}^{k}, \mathbf{b}_{i}\right)= \\
\left\{\begin{array}{l}
\left\|{ }_{W}^{C T} \mathbf{T} \boldsymbol{p}^{k}-\boldsymbol{b}_{i}^{s}\right\|^{2} \quad \text { if } \alpha<0 \\
\left\|_{W}^{C T} \mathbf{T} \boldsymbol{p}^{k}-\boldsymbol{b}_{i}^{e}\right\|^{2} \\
\left\|W \boldsymbol{p}^{C T}-\boldsymbol{b}_{i}^{s}\right\|^{2}-\alpha^{2} \text { otherwise }
\end{array}\right. \\
\alpha=\frac{\left({ }_{W}^{C T} \mathbf{T} \boldsymbol{p}^{k}-\boldsymbol{b}_{i}^{s}\right) \cdot\left(\boldsymbol{b}_{i}^{e}-\boldsymbol{b}_{i}^{s}\right)}{\left\|\boldsymbol{b}_{i}^{e}-\boldsymbol{b}_{i}^{s}\right\|},
\end{gathered}
$$

(Fig. 2) For all bronchial branches $\mathbf{b}_{i} \in \mathcal{B}$, we compute Eq. (2) and find the bronchial branch that minimizes Eq. (2) by

$$
\tilde{\mathbf{b}}=\arg \min _{\mathbf{b}_{i} \in \mathcal{B}} D\left({ }_{W}^{C T} \mathbf{T}^{l} \boldsymbol{p}^{k}, \mathbf{b}_{i}\right),
$$

If the RB tip exists around branching points, there is a possibility that there are multiple branches whose distance between ${ }_{W}^{C T} \mathbf{T}^{l} \boldsymbol{p}^{k}$ and them are equal or almost same. Therefore, we determine a unique correspondence between ${ }_{W}^{C T} \mathbf{T}^{l} \boldsymbol{p}^{k}$ and a bronchial branch by finding a branch $\mathbf{b}_{*}$ from $\boldsymbol{d}^{k}$ of the UEMT. First, we find a set of bronchial branches $\Phi$

$$
\Phi= \begin{cases}\mathcal{P}(\tilde{\mathbf{b}}) \cup \mathcal{C}(\mathcal{P}(\tilde{\mathbf{b}})) & \alpha<\gamma \times\left\|\tilde{\boldsymbol{b}}^{e}-\tilde{\boldsymbol{b}}^{s}\right\| \\ \{\tilde{\mathbf{b}}\} & \text { otherwise }\end{cases}
$$

from the results of Eq.(3). Then, for $\mathbf{b}_{j} \in \Phi$, we calculate the inner product of the running direction of $\mathbf{b}_{j}=\left\{\boldsymbol{b}_{j}^{s}, \boldsymbol{b}_{j}^{e}\right\}$ and ${ }_{W}^{C T} \mathbf{T}^{l} \boldsymbol{d}^{k}$ and find $\mathbf{b}_{*}$ showing the biggest value. This process can be expressed as

$$
\mathbf{b}_{*}=\arg \max _{\mathbf{b}_{j} \in \Phi} \frac{\left({ }_{W}{ }^{C} \mathbf{T}^{l} \boldsymbol{d}^{k}\right) \cdot\left(\boldsymbol{b}_{j}^{e}-\boldsymbol{b}_{j}^{s}\right)}{\left\|\boldsymbol{b}_{j}^{e}-\boldsymbol{b}_{j}^{s}\right\|} .
$$

This process means finding a bronchial branch $\mathbf{b}_{*}$ whose running direction is equal to the view direction of the RB from $\Phi$. 
(b) Calculation of corresponding point By using $\mathbf{b}_{*}$, we calculate the position $\boldsymbol{q}^{k} \in \mathcal{Q}$ on the medial lines of the bronchus corresponding to $\left(\boldsymbol{p}^{k}, \boldsymbol{d}^{k}\right) \in \mathcal{P}$ by

$$
\boldsymbol{q}^{k}=\boldsymbol{b}_{*}^{s}+\frac{\left({ }_{W}^{C T} \mathbf{T}^{l} \boldsymbol{p}^{k}-\boldsymbol{b}_{*}^{s}\right) \cdot\left(\boldsymbol{b}_{*}^{e}-\boldsymbol{b}_{*}^{s}\right)}{\left\|\boldsymbol{b}_{*}^{e}-\boldsymbol{b}_{*}^{s}\right\|} \times \frac{\left(\boldsymbol{b}_{*}^{e}-\boldsymbol{b}_{*}^{s}\right)}{\left\|\boldsymbol{b}_{*}^{e}-\boldsymbol{b}_{*}^{s}\right\|}
$$

(Fig. 2.) Also, we obtain the radius $r^{k}$ of the branch $\mathbf{b}_{*}$ corresponding to $\boldsymbol{q}^{k}$ and use a set of $r^{k}\left(\mathcal{R}=\left\{r^{k}\right\}\right)$ in the following process.

(c) Calculation of ${ }_{W}^{C T} \mathbf{T}$. By using $\left(\boldsymbol{p}^{k}, \boldsymbol{d}^{k}\right) \in \mathcal{P} . \boldsymbol{q}^{k} \in \mathcal{Q} . r^{k} \in \mathcal{R}$ obtained in the previous sections, we find the transformation matrix ${ }_{W}^{C T} \mathbf{T}$ minimizing

$$
\operatorname{Err}\left({ }_{W}^{C T} \mathbf{T}^{l}\right)=\sum_{\left(\boldsymbol{p}^{k}, \boldsymbol{d}^{k}\right) \in \mathcal{P}, \boldsymbol{q}^{k} \in \mathcal{Q}, r^{k} \in \mathcal{R}} \frac{\left\|\boldsymbol{q}^{k}-{ }_{W}^{C T} \mathbf{T}^{l} \boldsymbol{p}^{k}\right\|^{2}}{r^{k}},
$$

Powell method is utilized for this minimization process. Until the value of Eq. (8) does not change, we iterate the processes of (a)-(c).

Algorithm for ${ }_{W}^{C T} \mathbf{T}$ estimation. In this section, we show the actual algorithm for estimating ${ }_{W}^{C T} \mathbf{T}$. We estimate ${ }_{W}^{C T} \mathbf{T}$ for every $N_{1}$ measurements. In the following algorithm, we denote the number of updates of ${ }_{W}^{C T} \mathbf{T}$ as $l$ and ${ }_{W}^{C T} \mathbf{T}$ of the $l$-th estimation process as ${ }_{W}^{C T} \mathbf{T}^{l}$. Also, we need to perform Kitasaka's method [6] to segment bronchus regions from CT images and extract tree structures $\mathbf{b}_{i} \in \mathcal{B}$.

Estimation of ${ }_{W}^{C T} \mathbf{T}^{l}$

[Step 1] $l=1 . \mathcal{P}=\phi$

[Step 2] Obtain $N_{1}$ positions and orientation of the RB by 3.1 Let a set of positions and orientation be $\tilde{\mathcal{P}}=\left\{\left(\boldsymbol{p}^{n}, \boldsymbol{d}^{n}\right) ; n=1, \ldots, N_{1}\right\}$, then obtain $\mathcal{P}=\mathcal{P} \cup \tilde{\mathcal{P}}$.

[Step 3] If $|\mathcal{P}|<N_{2}$, go to Step2 Here, $|\mathcal{P}|$ shows the number of elements of a set $\mathcal{P}$.

[Step 4] $\mathbf{T}={ }_{W}^{C T} \mathbf{T}^{l-1}$.

[Step 5] For $\left(\boldsymbol{p}^{k}, \boldsymbol{d}^{k}\right) \in \mathcal{P}$, compute the position $\boldsymbol{q}^{k}$ on the medial lines. For all branches $\mathbf{b}_{i} \in \mathcal{B}$, find the branch $\tilde{\mathbf{b}}$ minimizing $D\left(\mathbf{T} \boldsymbol{p}^{k}, \mathbf{b}_{i}\right)$ of Eq. (2) by Eq. (4). Then, find the branch $\mathbf{b}_{*}$ corresponding to $\mathbf{T} \boldsymbol{p}^{k}$ by Eqs. (5) and (6) and compute $\boldsymbol{q}^{k}$ by Eq. (7) Let $\mathcal{Q}$ be a set of $\boldsymbol{q}^{k}$ and $\mathcal{R}$ be a set of the radius $r^{k}$ of the branch $\mathbf{b}_{*}$

[Step 6] From a pair of $\left(\boldsymbol{p}^{k}, \boldsymbol{d}^{k}\right) \in \mathcal{P}, \boldsymbol{q}^{k} \in \mathcal{Q}$ and $\boldsymbol{r}^{k} \in \mathcal{R}$ obtained in Step [5, find the transformation matrix $\mathbf{T}^{\prime}$ minimizing Eq. (8) by using $\mathbf{T}$ as the initial value.

[Step 7] Compute $\operatorname{Err}(\mathbf{T})$ and $\operatorname{Err}\left(\mathbf{T}^{\prime}\right)$ by Eq. (8). If $\operatorname{Err}\left(\mathbf{T}^{\prime}\right)<\operatorname{Err}(\mathbf{T})$, $\mathbf{T}=\mathbf{T}^{\prime}$ and go to Step 5 .

$\left[\right.$ Step 8] ${ }_{W}^{C T} \mathbf{T}^{l}=\mathbf{T}$,

[Step 9] Let $l$ be $l=l+1$ and go to Step 2 
(a)

(b)
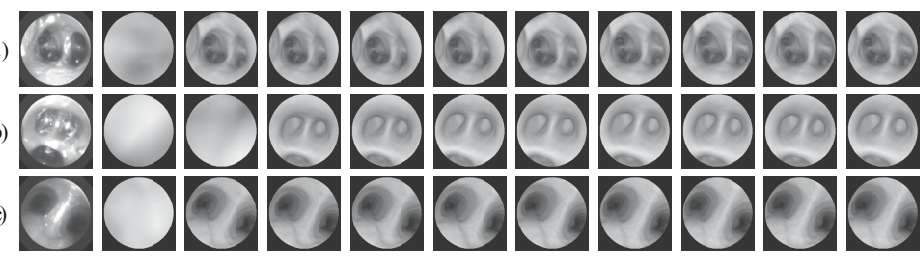

Fig. 4. VB images generated from the transformation matrix ${ }_{W}^{C T} \mathbf{T}^{l}$ with increasing update time $l$. (a) Trachea, (b) right lower lobe bronchus, and (c) left lower lobe bronchus.

\section{Experiments}

We evaluated the proposed method by using a rubber phantom of the bronchus fixed in an acrylic box. We inserted 24 pipes of $2 \mathrm{~mm}$ diameter in the box as shown in Fig. 3 and 18 pipes were used for accuracy evaluation. We took CT images of this phantom and extracted a bronchi region and its tree structure by Kitasaka's method 6]. Acquisition parameters of CT images are: $512 \times 512$ pixels, 341 slices, $0.684 \mathrm{~mm}$ of pixel spacing, $1.25 \mathrm{~mm}$ collimation and $0.5 \mathrm{~mm}$ reconstruction. We used BF-P260 (Olympus, Tokyo) as a bronchoscope, and 3DGuidance (Assention Technology Inc., Burlington, VT, U.S.A.) as the UEMT. We inserted the sensing coil of the UEMT through the working channel of the bronchoscope. The coil is fixed at the tip of the bronchoscope.

We inserted the RB into the bronchial branches of the phantom and estimated the transformation matrix ${ }_{W}^{C T} \mathbf{T}^{l}$. We obtained 2,000 pairs of RB images and UEMT outputs. Estimation of ${ }_{W}^{C T} \mathbf{T}^{l}$ is performed by using parameters of $N_{1}=20$. $N_{2}=200 . \gamma=0.2$. Initial estimation of ${ }_{W}^{C T} \mathbf{T}^{0}$ is determined from the orientation of the RB that is often taken at the trachea in actual bronchoscopy.

From three RB tip positions calculated from the UEMT outputs, we generated VB images by using ${ }_{W}^{C T} \mathbf{T}^{l}$. Also, with increasing $l$, we generated VB images. The results are shown in Fig. 4. By using the 18 pipes fixed in the acrylic box, we evaluated the estimation accuracy of ${ }_{W}^{C T} \mathbf{T}^{l}$. First, we inserted the UEMT into the pipe and measured the (RB) tip position $\boldsymbol{p}_{W}^{1} \ldots \boldsymbol{p}_{W}^{18}$. Then, we measured the position $\boldsymbol{p}_{C T}^{1} \ldots \boldsymbol{p}_{C T}^{18}$ on the CT images corresponding to $\boldsymbol{p}_{W}^{1} \ldots \boldsymbol{p}_{W}^{18}$ manually. We measured the registration error by

$$
E r r^{l}=\frac{1}{18} \sum_{k=1}^{18}\left\|\boldsymbol{p}_{C T}^{k}-{ }_{W}^{C T} \mathbf{T}^{l} \boldsymbol{p}_{W}^{k} \cdot\right\| .
$$

Here, $\boldsymbol{p}_{W}^{1} \ldots \boldsymbol{p}_{W}^{18}$ are measured 100 times under fixation of the UEMT, obtaining average values. Figure 5 shows the outputs of Eq. (9) obtained by the previous [4] and the proposed method with changing update times $l$.

To confirm the efficiency of the proposed method against breathing motion, we added virtual breathing motion to the outputs of the UEMT and estimated 


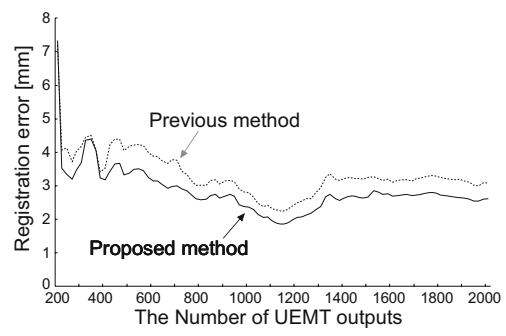

Fig. 5. Registration error of ${ }_{W}^{C T} \mathbf{T}^{l}$

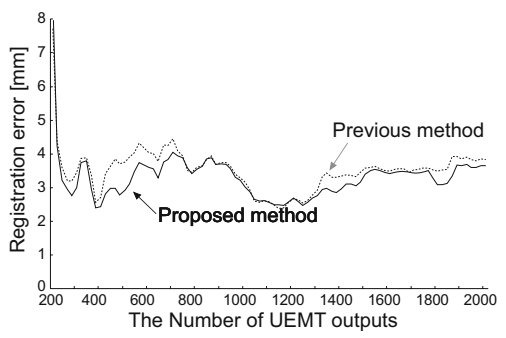

Fig. 6. Registration error of ${ }_{W}^{C T} \mathbf{T}^{l}$ with virtual breathing motion

${ }_{W}^{C T} \mathbf{T}^{l}$. In this virtual breathing motion, we set the displacement of the diaphragm as $\pm 20 \mathrm{~mm}$ of a sine curve and that of the trachea as 0 . The displacements of the rest parts are interpolated. This motion shows only head-to-foot motion. We performed the same accuracy assessment stated above (Fig. 6.)

\section{Discussion}

As presented in Fig. 5] the proposed method can decrease registration error of the transformation matrix ${ }_{W}^{C T} \mathbf{T}^{l}$ when the update time of ${ }_{W}^{C T} \mathbf{T}^{l}$ increases. The previous method showed the same tendency. The minimum registration errors of both methods are $1.86 \mathrm{~mm}$ for $l=48$ in the proposed method and $2.24 \mathrm{~mm}$ for $l=48$ in the previous method. Also, the update time $l$ when the registration error first becomes less than $3 \mathrm{~mm}$ was $l=27$ in the proposed method and $l=39$ in the previous method. From these results, the proposed method can obtain better accuracy with a smaller number of updates.

If the RB tip exists around branching points, there is a possibility that there are multiple breaches whose distance between ${ }_{W}^{C T} \mathbf{T}^{l} \boldsymbol{p}^{k}$ and them are equal or almost the same. However, the previous method used only the distance between the RB tip position ${ }_{W}^{C T} \mathbf{T} \boldsymbol{p}^{k}$ and the branches. Therefore, it was impossible to find unique correspondence between ${ }_{W}^{C T} \mathbf{T} \boldsymbol{p}^{k}$ and the branches. Sometimes, the transformation matrix minimizing Eq. (8) could not be found or took much computation time. In contrast, the proposed method utilizes the view direction of the RB camera obtained by the UEMT in finding the branch ${ }_{W}^{C T} \mathbf{T} \boldsymbol{p}^{k}$. This enables us to perform stable selection. Also, the diameter of a bronchial branch becomes smaller if we go to peripheral parts of the lung. This means the RB camera goes off from medial lines for thicker branches (such as the trachea.) Since the outputs of the UEMT go off from the medial lines for branches of larger diameter, this affected estimation of the transformation matrix. The proposed method solved this problem by introducing a normalization term in the minimization process.

As shown in Fig. 6, the proposed method showed good tracking performance even for the case of virtual breathing motion. Even though the UEMT outputs are altered by virtual breathing motion, the registration error is still under about $3 \mathrm{~mm}$. This is quite good registration performance in such a case. 
The proposed algorithm ran in real time. It took 0.065 sec. to update $\mathbf{T}$. Enough registration accuracy was obtained after traversing the main and the lower lobe bronchi of the left and the right lung.

\section{Conclusion}

This paper solved the problem of Deguchi's method [4 by utilizing the running direction information of the bronchus and the orientation information of the UEMT. The experimental results using a rubber phantom showed registration error was about $2.0 \mathrm{~mm}$. In the experiments generating virtual breathing motion, the registration error is still $3.0 \mathrm{~mm}$. The future work includes further investigation on effect of motion caused by other organs such as the heart.

Acknowledgments. The authors would like to thank Dr. H. Natori of Keiwakai Nishioka Hospital and Dr. M. Mori of Sapporo Kosei General Hospital for their advises. This work is supported by the program of the formation of innovation center for fusion of advanced technologies funded by the MEXT.

\section{References}

1. Mori, K., Deguchi, D., Sugiyama, J., et al.: Tracking of a bronchoscope using epipolar geometry analysis and intensity-based image registration of real and virtual endoscopic images. Med. Img. Ana. 6, 321-336 (2002)

2. Bricault, I., Ferretti, G., Cinquin, P.: Registration of Real and CT-Derived Virtual Bronchoscopic Images to Assist Transbronchial Biopsy. IEEE TMI 17(5), 703-714 (1998)

3. Wegner, K., Biederer, J., Tetzlaff, R., et al.: Evaluation and Extension of a Navigation System for Bronchoscopy inside Human Lungs. In: Proc. of SPIE, vol. 6509, pp. 65091H-1-12 (2007)

4. Deguchi, D., Ishitani, K., Kitasaka, T., et al.: A method for bronchoscope tracking using position sensor without fiducial markers. In: Proc, of SPIE, vol. 6511, pp. $65110 \mathrm{~N}-1-12(2007)$

5. Mori, K., Deguchi, D., Akiyama, K., et al.: Hybrid Bronchoscope Tracking Using a Magnetic Tracking Sensor and Image Registration. In: Duncan, J.S., Gerig, G. (eds.) MICCAI 2005. LNCS, vol. 3750, pp. 543-550. Springer, Heidelberg (2005)

6. Kitasaka, T., Mori, K., Hasegawa, J., et al.: A Method for Extraction of Bronchus Regions from 3D Chest X-ray CT Images by Analyzing Structural Features of the Bronchus. In: FORMA, vol. 17(4), pp. 321-338 (2002) 